\title{
Genetically Predicted Circulating Concentrations of Micronutrients and Risk of Amyotrophic Lateral Sclerosis: A Mendelian Randomization Study
}

\author{
Changqing $\mathrm{Mu}^{1 \dagger}$, Yating Zhao ${ }^{1 \dagger}$, Chen $\mathrm{Han}^{1 \dagger}$, Dandan Tian ${ }^{1}, \mathrm{Na} \mathrm{Guo}^{1}$, Chenguang Zhang ${ }^{1}$, \\ Ruixia Zhu ${ }^{1}$, Xiaoqian Zhang ${ }^{1}$, Jian Zhang ${ }^{2,3}$ and Xu Liu ${ }^{1 *}$ \\ ${ }^{1}$ Department of Neurology, First Affiliated Hospital of China Medical University, Shenyang, China, ${ }^{2}$ Department of Cell Biology, \\ Key Laboratory of Cell Biology, Ministry of Public Health, Shenyang, China, ${ }^{3}$ Key Laboratory of Medical Cell Biology, Ministry of \\ Education, China Medical University, Shenyang, China
}

Amyotrophic lateral sclerosis (ALS) is a progressive and devastating neurodegenerative disease with increasing incidence and high mortality, resulting in a considerable socioeconomic burden. Till now, plenty of studies have explored the potential relationship between circulating levels of various micronutrients and ALS risk. However, the observations remain equivocal and controversial. Thus, we conducted a two-sample Mendelian randomization (MR) study to investigate the causality between circulating concentrations of 9 micronutrients, including retinol, folate acid, vitamin B12, B6 and C, calcium, copper, zinc as well as magnesium, and ALS susceptibility. In our analysis, several single nucleotide polymorphisms were collected as instrumental variables from large-scale genome-wide association studies of these 9 micronutrients. Then, inverse variance weighted (IVW) approach as well as alternative MR-Egger regression, weighted median and MR-pleiotropy residual sum and outlier (MR-PRESSO) analyses were performed to evaluate causal estimates. The results from IMW analysis showed that there was no causal relationship of 9 micronutrients with ALS risk. Meanwhile, the three complementary approaches obtained similar results. Thus, our findings indicated that supplementation of these 9 micronutrients may not play a clinically effective role in preventing the occurrence of ALS.

Keywords: amyotrophic lateral sclerosis, micronutrient, mendelian randomization study, genome-wide association study, susceptibility

\section{INTRODUCTION}

Amyotrophic lateral sclerosis (ALS) is a fatal heterogeneous neurodegenerative disorder which selectively damages upper and lower motor neurons (van Es et al., 2017). It is typically characterized by progressive motor deficits such as dysphagia, dysarthria, muscle atrophy and weakness of the trunk and extremities, and even respiratory failure (Masrori and Van Damme, 2020). Globally, the number of individuals suffered from ALS reached 222,801 in 2015 and it is expected to constantly increase, reaching 376,674 by 2040 (Arthur et al., 2016). Moreover, the per capita cost related to ALS was the highest among various neurological diseases, and in the United States alone, the standardized total expense of ALS was estimated to range from \$279 to \$472 million (Gladman and Zinman, 2015). Due to severe clinical symptoms and huge socio-economic burden, an increasing number of 


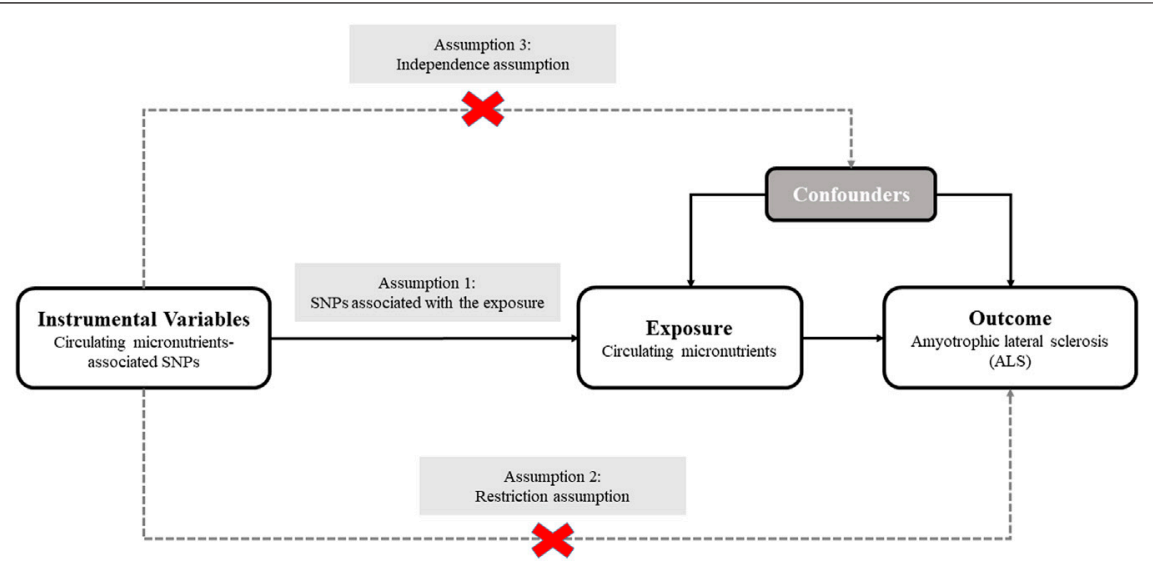

FIGURE 1 | The overall design of Mendelian randomization analysis in the present study.

studies have been implemented to investigate the predisposition factors of ALS and explore its possible pathogenesis.

In recent years, micronutrients including vitamins and minerals were found to be related to multiple neurodegenerative diseases such as $\mathrm{AD}$ and $\mathrm{PD}$ ( $\mathrm{Hu}$ et al., 2013; Boulos et al., 2019). Regarding ALS, the relationship between various micronutrients and ALS risk has not yet been determined. For example, a center-based survey including 202 ALS patients and 208 healthy controls showed that lower circulating levels of vitamin $\mathrm{C}$ and higher levels of retinol were significantly related to an increased risk of ALS (Wang et al., 2020). Moreover, Peters et al. observed a significant association between circulating zinc levels and ALS susceptibility based on a prospective cohort study involving 520,000 European participants (Peters et al., 2021). However, some other evidence indicated no statistically significant association between circulating levels of vitamin $\mathrm{C}$ as well as retinol and ALS risk (Iwasaki et al., 1995; Paraskevas et al., 1997). Similarly, a case-control study by Forte et al. failed to observe a difference in circulating zinc levels between ALS cases and healthy controls (Forte et al., 2017). Due to the small sample size, inevitable potential confounding factors and reverse causation, the results from the observational studies mentioned above are inconsistent.

For overcoming the conventional bias of observational studies, mendelian randomization (MR) analysis could reveal the causal relationship between predisposition factors and ALS susceptibility by applying single nucleotide polymorphisms (SNPs) as instrumental variables (IVs) as well as large-scale data from genome-wide association studies (GWASs). Indeed, three previous MR studies have revealed no causal effect of serum 25-hydroxyvitamin D, iron and selenium on ALS risk (Larsson and Roos, 2020; Cai et al., 2021; He and Cui, 2021). However, up till now, apart from these three micronutrients, no systematic MR analysis has been published for other vitamins and minerals. Therefore, we conducted this two-sample MR analysis to more accurately infer the causal relationship between circulating levels of various micronutrients and ALS risk.

\section{MATERIALS AND METHODS}

\section{Study Design, Instruments Selection and Data Sources}

We designed a two-sample MR analysis to investigate the causal relationship of plasma micronutrients on ALS (Pierce and Burgess, 2013). The complete two-sample design of our MR framework is displayed in Figure 1. For determining interesting exposures, a search for the published GWASs on the circulating levels of various micronutrients was conducted using PubMed databases. A total of 7 GWASs involving 9 micronutrients were identified, including 5 vitamins (retinol, folate acid, vitamin B12, B6 and C) and 4 minerals (calcium, copper, zinc and magnesium) (Hazra et al., 2009; Meyer et al., 2010; Mondul et al., 2011; Evans et al., 2013; Grarup et al., 2013; O'Seaghdha et al., 2013; Zheng et al., 2021). The detailed information of GWASs related to 9 exposures are listed in Table 1. Next, the candidate SNPs representing the circulating levels of 9 micronutrients were selected by a genome-wide significance threshold $(p<5.00 \mathrm{E}-08)$. Lastly, we used a $r^{2}$ threshold $<0.01$ to prune all selected SNPs, which retaining 43 SNPs with the lowest $p$-value for detecting relationship between circulating levels of 9 micronutrients and ALS risk. Regarding GWAS dataset for ALS susceptibility, summary association statistics was collected from the most recent and comprehensive GWAS, which totally included 80,610 individuals of European descent (20,806 ALS cases and 59,804 controls) (Nicolas et al., 2018). For these 43 selected SNPs, $\beta$ coefficients and standard errors for effect of SNPs on 9 micronutrients and ALS risk were extracted from abovementioned GWAS datasets for subsequent analysis (Supplementary Table S1). Since we only retrieved GWAS summary-level data rather than individual-level data, no additional ethical permit was required.

\section{Statistical Analyses}

In order to investigate the potential causal relationship between circulating levels of 9 micronutrients and ALS risk, two-sample 
TABLE 1 | Summary of details on GWASs and related datasets involving nine micronutrients in our study.

\begin{tabular}{|c|c|c|c|c|}
\hline Exposures & Cohorts or datasets & Participants & Publicly available websites & PMID \\
\hline Vitamin C & $\begin{array}{l}\text { Fenland study, EPIC InterAct study, EPIC Norfolk study, EPIC-CVD } \\
\text { study }\end{array}$ & $\begin{array}{l}52,018 \text { individuals of European } \\
\text { ancestry }\end{array}$ & $\begin{array}{l}\text { https://doi.org/10.6084/m9. } \\
\text { figshare.13227443.v1 }\end{array}$ & 33203707 \\
\hline Vitamin B12 & Icelanders, Danish-Inter99, Danish-Health2006 & $\begin{array}{l}45,576 \text { individuals of European } \\
\text { ancestry }\end{array}$ & NA & 23754956 \\
\hline Folate acid & Icelanders, Danish-Inter99, Danish-Health2006 & $\begin{array}{l}37,341 \text { individuals of European } \\
\text { ancestry }\end{array}$ & NA & 23754956 \\
\hline Retinol & ATBC study, PLCO study, NHS studies, InCHIANTI & $\begin{array}{l}8,902 \text { individuals (mostly } \\
\text { European ancestry) }\end{array}$ & NA & 21878437 \\
\hline Vitamin B6 & CGEMS study, SHARe study & $\begin{array}{l}4,763 \text { individuals of European } \\
\text { ancestry }\end{array}$ & NA & 19744961 \\
\hline Calcium & $\begin{array}{l}\text { AGES, ARIC study, BLSA, CoLAUS, CROATIA-Vis, CROATIA-Korcula, } \\
\text { CROATIA-Split, FHS, HABC, InCHIANTI, LBC 1936, LOLIPOP EW A, } \\
\text { LOLIPOP EW P, LOLIPOP EW610, OGP Talana, ORCADES, SHIP, } \\
\text { RS, CHS }\end{array}$ & $\begin{array}{l}39,400 \text { individuals of European } \\
\text { ancestry }\end{array}$ & NA & 24068962 \\
\hline Magnesium & ARIC study, FHS, RS & $\begin{array}{l}\text { 15,366 individuals of European } \\
\text { ancestry }\end{array}$ & NA & 20700443 \\
\hline Copper & QIMR studies & $\begin{array}{l}2,603 \text { individuals of European } \\
\text { ancestry }\end{array}$ & $\begin{array}{l}\text { https://genepi.qimr.edu.au/ } \\
\text { general/downloadable.cgi }\end{array}$ & 23720494 \\
\hline Zinc & QIMR studies & $\begin{array}{l}\text { 2,603 individuals of European } \\
\text { ancestry }\end{array}$ & $\begin{array}{l}\text { https://genepi.qimr.edu.au/ } \\
\text { general/downloadable.cgi }\end{array}$ & 23720494 \\
\hline
\end{tabular}

MR analysis was performed by employing five statistical methods including Wald method, inverse-variance weighted (IVW), MREgger, weighted median and MR-Pleiotropy Residual Sum and Outlier (MR-PRESSO). In detail, we applied Wald method to evaluate the causal relationship for the micronutrient with only one related SNP as IV. If more than one SNP involved, IVW method was implemented as the primary analysis to explore the causal effect on ALS risk. Moreover, the Cochran's $Q$ and funnel plot test was used to evaluate the heterogeneity of the causal estimates among multiple SNPs. Subsequent leave-one-out sensitivity analysis was performed to investigate whether a single SNP was driving the IVW point estimate.

Additionally, in the IVW MR analysis, the ratios of SNPmicronutrients to SNP-ALS were combined in a meta-analysis to explore the causal relationship between circulating levels of micronutrients and ALS risk (Burgess et al., 2013). The premise of IVW analysis is that all SNPs are valid IVs, and it could lead to an increased statistical power and accurate estimate when the core assumption of MR is satisfied. However, if the genetic variants affect ALS susceptibility through other pathways than micronutrients (horizontal pleiotropy), the causal estimate may be biased (Hemani et al., 2018). Therefore, we conducted a complementary analysis by using the weighted median and MREgger and MR-PRESSO methods. Specifically, weighted median method could maintain reliable estimates even if up to half of IVs are invalid (Bowden et al., 2016). Regarding MR-Egger regression method, it does not limit the slope to pass zero in the micronutrients-ALS causal estimate and identifies the presence of potential pleiotropy by calculating the intercept value (Bowden et al., 2015). Besides, the MR-PRESSO global and outlier test was also implemented as an approach for detecting pleiotropic outliers (Verbanck et al., 2018).

Given 9 exposures involved in our MR analysis, we set the statistically significant threshold of $p$-value to 5.56E-03 (0.05/9) according to Bonferroni correction. Meanwhile, a $p$-value, which implied the suggestive evidence for a possible causal role, was set to range from 5.56E-03 to 0.05 . All above analyses were performed using $\mathrm{R}$ version 4.0.3 software with R-packages (MendelianRandomization, TwoSampleMR and MR-PRESSO).

\section{RESULTS}

\section{Circulating Levels of Vitamins and ALS Risk} As shown in Figure 2 and Supplementary Figure S1, the MR estimates obtained by the IVW method suggested that predicted circulating concentrations of four vitamins including vitamin $\mathrm{C}$ $(\mathrm{OR}=0.89,95 \%$ CI: 0.75 to $1.05, p=0.163)$, vitamin $\mathrm{B} 12(\mathrm{OR}=$ $0.99,95 \%$ CI: 0.90 to $1.08, p=0.756)$, folate acid (OR $=0.88,95 \%$ CI: 0.63 to $1.24, p=0.281)$ and retinol $(\mathrm{OR}=1.25,95 \% \mathrm{CI}: 0.66$ to $2.39, p=0.493$ ) were causally unrelated to ALS susceptibility. In addition, for each vitamin, we did not observe any obvious heterogeneity through the Cochran's $Q$ test $(p>0.05)$. However, regarding vitamin $C$, the visual inspection of the funnel plot showed slight asymmetry and potential heterogeneity (Supplementary Figure S2). When leave-one-out sensitivity analysis was performed, the change of IVW point estimate was detected for vitamin C when omitting rs2559850, but not for vitamin B12 (Supplementary Figure S3). Moreover, 3 additional alternative approaches were also applied containing 


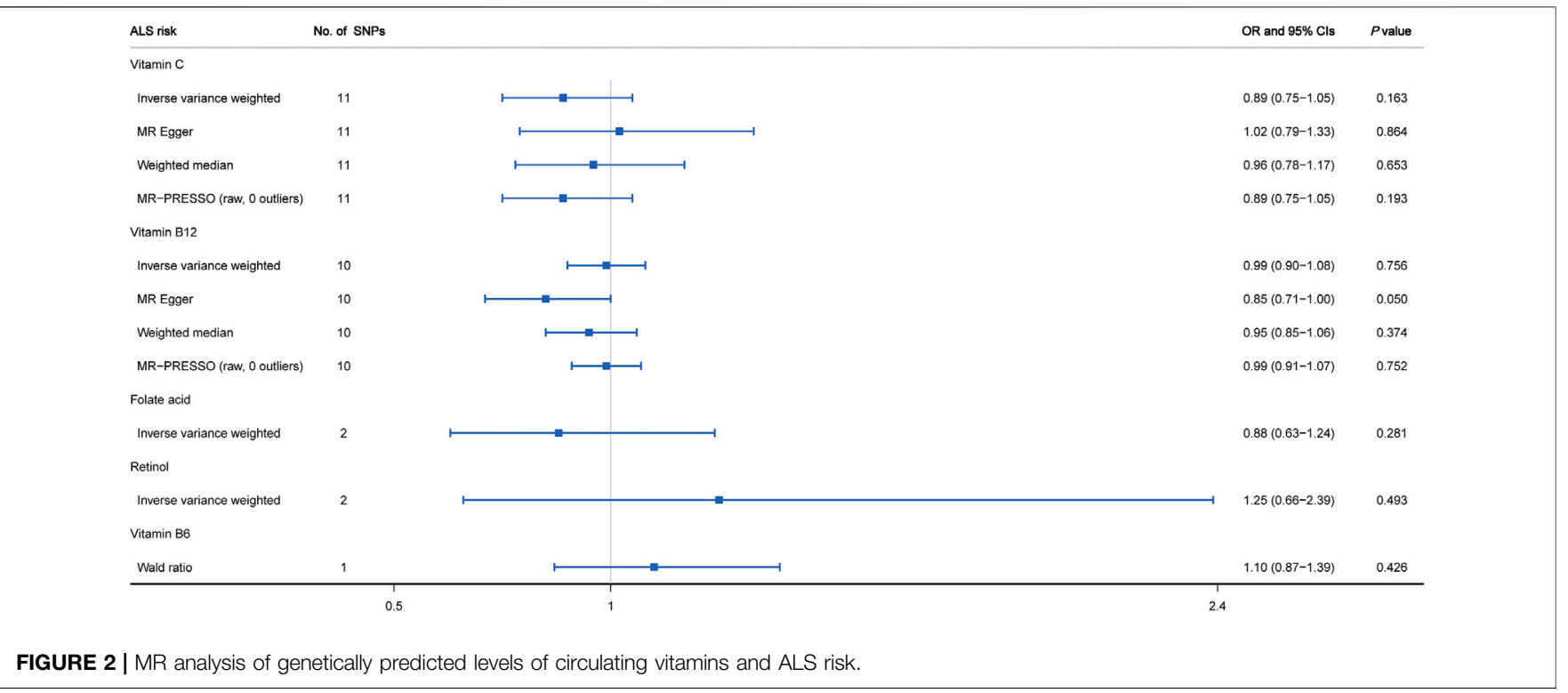

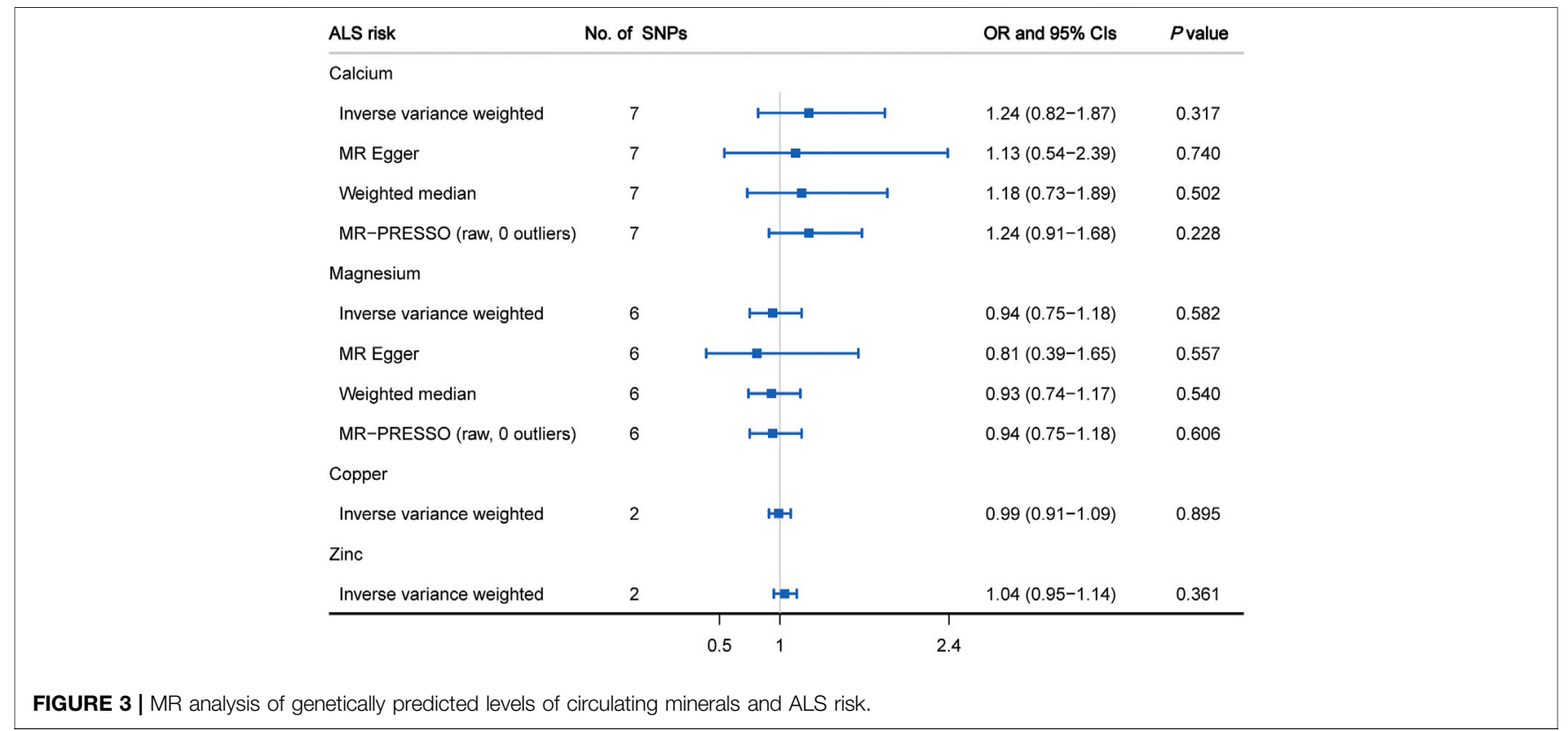

MR-Egger, weighted median and MR-PRESSO. The results further supported that there was no causal relationship between vitamin $\mathrm{C}$ as well as vitamin B12 and ALS risk (Figure 2). Based on the MREgger regression analysis, we did not observe evidence of potential pleiotropy between circulating levels of vitamin C and ALS risk (intercept $=-0.012, p=0.173$ ). However, a mild pleiotropy could be detected for vitamin $\mathrm{B} 12$ (intercept $=0.024, p=0.036$ ). In addition, no potential SNP outliers were identified by MR-PRESSO test (vitamin C: $p=0.249$; vitamin B12: $p=0.538$ ). Lastly, regarding vitamin B6 with only one related SNP as IV, by using the Wald ratio method, we did not find any causal effect on ALS risk $(\mathrm{OR}=$ 1.10; 95\% CI: 0.87-1.39; $p=0.426$ ).
Circulating Levels of Minerals and ALS Risk

Figure 3 displays the causal estimates of four minerals on ALS risk. In the primary analysis using IVW method, we did not observe any causal relationship between circulating levels of calcium $(\mathrm{OR}=1.24,95 \% \mathrm{CI}: 0.82$ to $1.87, p=0.317)$, magnesium ( $\mathrm{OR}=0.94,95 \% \mathrm{CI}: 0.75$ to $1.18, p=0.582)$, copper (OR $=0.99,95 \% \mathrm{CI}: 0.91$ to $1.09, p=0.895)$, zinc (OR $=1.04,95 \%$ CI: 0.95 to $1.14, p=0.361)$ and ALS occurrence (Supplementary Figure S4). Meanwhile, there was no significant heterogeneity measured by Cochran's $Q$ test $(p>0.05)$ and funnel plot (Supplementary Figure S2). Subsequent sensitivity analysis involving calcium and magnesium showed that no single SNP 
dominated the IVW point estimate (Supplementary Figure S3). Regarding calcium and magnesium, the results in IVW analysis were further verified by using MR- Egger, weighted median and MR-PRESSO methods (Figure 3). Additionally, no significant pleiotropic effects for included SNPs were detected by MR-Egger regression test (calcium: intercept $=0.003, p=0.789$; magnesium: intercept $=0.011, p=0.659$ ). In the subsequent analysis using MR-PRESSO method, we did not find any outliers for these two minerals (calcium: $p=0.829$; magnesium: $p=0.183$ ).

\section{DISCUSSION}

In spite of the short survival time and the increased incidence of ALS, unfortunately, no effective preventions have been found so far (Traxinger et al., 2013; Xu et al., 2020). Thus, for further exploring possible preventive measures, MR analysis was introduced to investigate whether 9 micronutrients were causally related to ALS risk or not. Here, we did not find any causal relationship between 9 micronutrients and ALS susceptibility, including 5 vitamins (retinol, folate acid, vitamin B12, B6 and C) and 4 minerals (calcium, copper, zinc and magnesium).

Recently, an increasing number of studies showed that high homocysteine levels were closely associated with a variety of pathological processes, including apoptosis and autophagy, mitochondrial dysfunction and oxidative stress, thereby damaging motor neurons and leading to ALS occurrence (Zoccolella et al., 2010). Moreover, vitamin B6, B12 and folate acid would change homocysteine status by mediating one-carbon metabolism (Hao et al., 2007; Clare et al., 2019). Thus, a series of experimental studies have explored whether these three B vitamins can participate in ALS occurrence. For example, a cellular study by Hemendinger et al. (2011) reported vitamin B12 could block the apoptosis of motor neuron-like cells by reducing homocysteine levels while methylfolate could not. Meanwhile, in the SOD1G93A transgenic mouse model of ALS, folate acid alone or a combination of folate acid and vitamin B12 exhibited neuroprotective effects on motor neurons and retarded the onset of ALS (Zhang et al., 2008). More importantly, several observational studies have also been performed to investigate the association between B vitamins and ALS susceptibility. In 2007, Izumi et al. observed that the mean survival time of 18 patients in the vitamin B12 treatment group was significantly longer than that of 16 patients without any treatment (Izumi and Kaji, 2007). Conversely, a randomized controlled study including 373 Japanese ALS patients showed that intramuscular injection of high-dose vitamin B12 had no significant effect on the development of ALS (Kaji et al., 2019). Regarding vitamin C and retinol, as two of the most common antioxidants, they may have the potential to delay the initiation and progression of ALS by reducing oxidative stress (Esposito et al., 2002). In previous experiments on familial ALS model mice, no significant difference in the age of ALS onset was detected between the vitamin C treatment group and control group, while long-term supplementation of retinoic acid would shorten the lifespan of ALS mice (Nagano et al., 2003; Crochemore et al., 2009). However, a case-control study involving 40 ALS patients and 87 healthy controls did not support the association between circulating retinol levels and ALS risk (Molina et al., 1999). Additionally, in a meta-analysis of five large cohorts including 1,053,575 participants, Fitzgerald et al. reported that high dietary intake of vitamin $\mathrm{C}$ was not related to ALS susceptibility (Fitzgerald et al., 2013). Overall, the discrepant results of these studies on 5 vitamins aforementioned may be due to the small sample size, limited follow-up time, confounding and reverse causality. Thus, we introduced the more reliable MR analysis and finally inferred that there was no causal relationship between genetically predicted circulating levels of folate acid, vitamin $\mathrm{B} 12, \mathrm{~B} 6$, and $\mathrm{C}$, retinol and the risk of ALS.

Regarding the four minerals analyzed in this study, many observational studies focusing on their relationship with ALS susceptibility have been performed. However, until now, the results of these studies were inconsistent and inconclusive. For instance, in a case-control study involving 392 participants, Peters et al. (2016) observed the statistically significant association between copper and zinc and ALS susceptibility. Nevertheless, in other case-control studies, these association cannot be replicated (Roos et al., 2013; Forte et al., 2017). Thus, we have introduced the MR analysis, hoping to provide a broader and more reliable perspective on the causal relationship between 4 minerals and ALS risk. Here, our results did not support that genetically predicted circulating levels of calcium, copper, zinc and magnesium were causally related to the occurrence of ALS.

Several advantages of our study were as follows. First, this is the first MR analysis for determining the causal relationship between various micronutrients including 5 vitamins as well as 4 minerals and ALS risk. Second, we adopted large-scale GWAS datasets involving thousands of participants to ensure the robustness and authenticity of our MR analysis. Third, for all micronutrients, no heterogeneity was measured between SNPs by Cochran's $Q$ test. Moreover, we chose three complementary methods, including MREgger, weighted median and MR-PRESSO, which further confirmed most of the results from IVW analysis. Some limitations should be noted in the present MR analysis. First, a mild pleiotropy for vitamin B12 can be detected using MR-Egger method. Meanwhile, in the leave-one-out analysis for vitamin C, we found that omission of rs2559850 had slight influence on the causal relationship. Moreover, the funnel plot of vitamin $\mathrm{C}$ indicated potential heterogeneity. Thus, the note should be taken when interpreting the relationship of vitamin B12 and C with ALS risk. Second, almost all participants of GWAS datasets in our analysis were Europeans, which limited the general application in other populations. Third, since the data involving the clinical characteristics of ALS patients were not available, such as age, gender, age at onset and disease progression, etc., we can only analyze the potential association between micronutrients and ALS susceptibility. 


\section{CONCLUSION}

In conclusion, this MR study provided no evidence for causal association between genetically predicted circulating levels of 9 micronutrients and ALS risk. These findings indicate that supplementation of these 9 micronutrients might not be clinically helpful in preventing the occurrence of ALS.

\section{DATA AVAILABILITY STATEMENT}

The datasets used and/or analyzed during the current study are available from the corresponding author on reasonable request.

\section{AUTHOR CONTRIBUTIONS}

$\mathrm{XL}$ designed the research. $\mathrm{CM}$ and $\mathrm{CH}$ had full access to all the data in the study and take responsibility for the integrity of the data and the accuracy of the data analysis. CM and YZ wrote the paper and performed the data analysis. All authors contributed to the statistical analysis, critically reviewed the manuscript during the writing process, and approved the final version to be published.

\section{REFERENCES}

Arthur, K. C., Calvo, A., Price, T. R., Geiger, J. T., Chiò, A., and Traynor, B. J. (2016). Projected Increase in Amyotrophic Lateral Sclerosis from 2015 to 2040. Nat. Commun. 7, 12408. doi:10.1038/ncomms 12408

Boulos, C., Yaghi, N., El Hayeck, R., Heraoui, G. N., and Fakhoury-Sayegh, N. (2019). Nutritional Risk Factors, Microbiota and Parkinson's Disease: What Is the Current Evidence? Nutrients 11 (8), 1896. doi:10.3390/nu11081896

Bowden, J., Davey Smith, G., and Burgess, S. (2015). Mendelian Randomization with Invalid Instruments: Effect Estimation and Bias Detection through Egger Regression. Int. J. Epidemiol. 44 (2), 512-525. doi:10.1093/ije/dyv080

Bowden, J., Davey Smith, G., Haycock, P. C., and Burgess, S. (2016). Consistent Estimation in Mendelian Randomization with Some Invalid Instruments Using a Weighted Median Estimator. Genet. Epidemiol. 40 (4), 304-314. doi:10.1002/ gepi.21965

Burgess, S., Butterworth, A., and Thompson, S. G. (2013). Mendelian Randomization Analysis with Multiple Genetic Variants Using Summarized Data. Genet. Epidemiol. 37 (7), 658-665. doi:10.1002/gepi.21758

Cai, J., Chen, X., Wang, H., Wei, Z., Li, M., Rong, X., et al. (2021). Iron Status May Not Affect Amyotrophic Lateral Sclerosis: A Mendelian Randomization Study. Front. Genet. 12, 617245. doi:10.3389/fgene.2021.617245

Clare, C. E., Brassington, A. H., Kwong, W. Y., and Sinclair, K. D. (2019). OneCarbon Metabolism: Linking Nutritional Biochemistry to Epigenetic Programming of Long-Term Development. Annu. Rev. Anim. Biosci. 7, 263-287. doi:10.1146/annurev-animal-020518-115206

Crochemore, C., Virgili, M., Bonamassa, B., Canistro, D., Pena-Altamira, E., Paolini, M., et al. (2009). Long-term Dietary Administration of Valproic Acid Does Not Affect, while Retinoic Acid Decreases, the Lifespan of G93A Mice, a Model for Amyotrophic Lateral Sclerosis. Muscle Nerve 39 (4), 548-552. doi: $10.1002 /$ mus. 21260

Esposito, E., Rotilio, D., Dimatteo, V., Digiulio, C., Cacchio, M., and Algeri, S. (2002). A Review of Specific Dietary Antioxidants and the Effects on Biochemical Mechanisms Related to Neurodegenerative Processes. Neurobiol. Aging 23 (5), 719-735. doi:10.1016/s0197-4580(02)00078-7

\section{FUNDING}

This work was supported by the National Natural Science Foundation of China (Grant No. 81400950, 81801053, 81872044), Natural Science Foundation of Liaoning Province (Grant No. 2019-MS-365).

\section{SUPPLEMENTARY MATERIAL}

The Supplementary Material for this article can be found online at: https://www.frontiersin.org/articles/10.3389/fgene.2021.811699/ full\#supplementary-material

Supplementary Table 1 | Characteristics of the SNPS associated with circulating micronutrients and their association with ALS.

Supplementary Figure 1 | Forest plots of the causal effects of vitamins-associated SNPS on ALS risk. (A) vitamin C, (B) vitamin B12, (C) Folate acid, (D): Retinol.

Supplementary Figure 2 | Funnel plots of the causal effects of micronutrientsassociated SNPs on ALS risk. (A) vitamin C, (B) vitamin B12, (C) Calcium, (D) Magnesium.

Supplementary Figure $\mathbf{3}$ | Sensitivity analyses of the causal effects of micronutrients-associated SNPs on ALS risk. (A) vitamin C, (B) vitamin B12, (C) Calcium, (D) Magnesium.

Supplementary Figure 4 | Forest plots of the causal effects of minerals-associated SNPs on ALS risk. (A) Calcium, (B) Magnesium, (C) Copper, (D) Zinc.

Evans, D. M., Zhu, G., Dy, V., Heath, A. C., Madden, P. A. F., Kemp, J. P., et al. (2013). Genome-wide Association Study Identifies Loci Affecting Blood Copper, Selenium and Zinc. Hum. Mol. Genet. 22 (19), 3998-4006. doi:10.1093/hmg/ddt239

Fitzgerald, K. C., O’Reilly, É. J., Fondell, E., Falcone, G. J., McCullough, M. L., Park, Y., et al. (2013). Intakes of Vitamin C and Carotenoids and Risk of Amyotrophic Lateral Sclerosis: Pooled Results from 5 Cohort Studies. Ann. Neurol. 73 (2), 236-245. doi:10.1002/ana.23820

Forte, G., Bocca, B., Oggiano, R., Clemente, S., Asara, Y., Sotgiu, M. A., et al. (2017). Essential Trace Elements in Amyotrophic Lateral Sclerosis (ALS): Results in a Population of a Risk Area of Italy. Neurol. Sci. 38 (9), 1609-1615. doi:10.1007/ s10072-017-3018-2

Gladman, M., and Zinman, L. (2015). The Economic Impact of Amyotrophic Lateral Sclerosis: a Systematic Review. Expert Rev. Pharmacoecon. Outcomes Res. 15 (3), 439-450. doi:10.1586/14737167.2015.1039941

Grarup, N., Sulem, P., Sandholt, C. H., Thorleifsson, G., Ahluwalia, T. S., Steinthorsdottir, V., et al. (2013). Genetic Architecture of Vitamin B12 and Folate Levels Uncovered Applying Deeply Sequenced Large Datasets. Plos Genet. 9 (6), e1003530. doi:10.1371/journal.pgen.1003530

Hao, L., Ma, J., Zhu, J., Stampfer, M. J., Tian, Y., Willett, W. C., et al. (2007). High Prevalence of Hyperhomocysteinemia in Chinese Adults Is Associated with Low Folate, Vitamin B-12, and Vitamin B-6 Status. J. Nutr. 137 (2), 407-413. doi:10.1093/jn/137.2.407

Hazra, A., Kraft, P., Lazarus, R., Chen, C., Chanock, S. J., Jacques, P., et al. (2009). Genome-wide Significant Predictors of Metabolites in the One-Carbon Metabolism Pathway. Hum. Mol. Genet. 18 (23), 4677-4687. doi:10.1093/hmg/ddp428

He, D., and Cui, L. (2021). Assessing the Causal Role of Selenium in Amyotrophic Lateral Sclerosis: A Mendelian Randomization Study. Front. Genet. 12, 724903. doi:10.3389/fgene.2021.724903

Hemani, G., Bowden, J., and Davey Smith, G. (2018). Evaluating the Potential Role of Pleiotropy in Mendelian Randomization Studies. Hum. Mol. Genet. 27 (R2), R195-R208. doi:10.1093/hmg/ddy163

Hemendinger, R. A., Armstrong, E. J., 3rd, and Brooks, B. R. (2011). Methyl Vitamin B12 but Not Methylfolate Rescues a Motor Neuron-like Cell Line from Homocysteine-Mediated Cell Death. Toxicol. Appl. Pharmacol. 251 (3), 217-225. doi:10.1016/j.taap.2011.01.003 
Hu, N., Yu, J.-T., Tan, L., Wang, Y.-L., Sun, L., and Tan, L. (2013). Nutrition and the Risk of Alzheimer's Disease. Biomed. Res. Int. 2013, 1-12. doi:10.1155/2013/524820

Iwasaki, Y., Ikeda, K., and Kinoshita, M. (1995). Vitamin A and E Levels Are normal in Amyotrophic Lateral Sclerosis. J. Neurol. Sci. 132 (2), 193-194. doi:10.1016/0022-510x(95)00145-r

Izumi, Y., and Kaji, R. (2007). Clinical Trials of Ultra-high-dose Methylcobalamin in ALS. Brain Nerve 59 (10), 1141-1147. doi:10.11477/mf.1416100154

Kaji, R., Imai, T., Iwasaki, Y., Okamoto, K., Nakagawa, M., Ohashi, Y., et al. (2019). Ultra-high-dose Methylcobalamin in Amyotrophic Lateral Sclerosis: a LongTerm Phase II/III Randomised Controlled Study. J. Neurol. Neurosurg. Psychiatry 90 (4), 451-457. doi:10.1136/jnnp-2018-319294

Larsson, S. C., and Roos, P. M. (2020). Serum 25-hydroxyvitamin D in Amyotrophic Lateral Sclerosis: Mendelian Randomization Study. Neurobiol. Aging 87, 140.e1-140.e3. doi:10.1016/j.neurobiolaging.2019.10.024

Masrori, P., and Van Damme, P. (2020). Amyotrophic Lateral Sclerosis: a Clinical Review. Eur. J. Neurol. 27 (10), 1918-1929. doi:10.1111/ene.14393

Meyer, T. E., Verwoert, G. C., Hwang, S.-J., Glazer, N. L., Smith, A. V., van Rooij, F. J. A., et al. (2010). Genome-wide Association Studies of Serum Magnesium, Potassium, and Sodium Concentrations Identify Six Loci Influencing Serum Magnesium Levels. Plos Genet. 6 (8), e1001045. doi:10.1371/journal.pgen.1001045

Molina, J. A., Bustos, F., Jiménez-Jiménez, F. J., Esteban, J., Guerrero-Sola, A., Zurdo, M., et al. (1999). Serum Levels of Beta-Carotene, Alpha-Carotene, and Vitamin A in Patients with Amyotrophic Lateral Sclerosis. Acta Neurol. Scand. 99 (5), 315-317. doi:10.1111/j.1600-0404.1999.tb00682.x

Mondul, A. M., Yu, K., Wheeler, W., Zhang, H., Weinstein, S. J., Major, J. M., et al. (2011). Genome-wide Association Study of Circulating Retinol Levels. Hum. Mol. Genet. 20 (23), 4724-4731. doi:10.1093/hmg/ddr387

Nagano, S., Fujii, Y., Yamamoto, T., Taniyama, M., Fukada, K., Yanagihara, T., et al. (2003). The Efficacy of Trientine or Ascorbate Alone Compared to that of the Combined Treatment with These Two Agents in Familial Amyotrophic Lateral Sclerosis Model Mice. Exp. Neurol. 179 (2), 176-180. doi:10.1016/s00144886(02)00014-6

Nicolas, A., Kenna, K. P., Renton, A. E., Ticozzi, N., Faghri, F., Chia, R., et al. (2018). Genome-wide Analyses Identify KIF5A as a Novel ALS Gene. Neuron 97 (6), 1268-1283.e6. doi:10.1016/j.neuron.2018.02.027

O'Seaghdha, C. M., Wu, H., Yang, Q., Kapur, K., Guessous, I., Zuber, A. M., et al. (2013). Meta-analysis of Genome-wide Association Studies Identifies Six New Loci for Serum Calcium Concentrations. Plos Genet. 9 (9), e1003796. doi:10.1371/journal.pgen.1003796

Paraskevas, G. P., Kapaki, E., Libitaki, G., Zournas, C., Segditsa, I., and Papageorgiou, C. (1997). Ascorbate in Healthy Subjects, Amyotrophic Lateral Sclerosis and Alzheimer's Disease. Acta Neurol. Scand. 96 (2), 88-90. doi:10.1111/j.1600-0404.1997.tb00245.x

Peters, S., Broberg, K., Gallo, V., Levi, M., Kippler, M., Vineis, P., et al. (2021). Blood Metal Levels and Amyotrophic Lateral Sclerosis Risk: A Prospective Cohort. Ann. Neurol. 89 (1), 125-133. doi:10.1002/ana.25932

Peters, T. L., Beard, J. D., Umbach, D. M., Allen, K., Keller, J., Mariosa, D., et al. (2016). Blood Levels of Trace Metals and Amyotrophic Lateral Sclerosis. Neurotoxicology 54, 119-126. doi:10.1016/j.neuro.2016.03.022

Pierce, B. L., and Burgess, S. (2013). Efficient Design for Mendelian Randomization Studies: Subsample and 2-sample Instrumental Variable Estimators. Am. J. Epidemiol. 178 (7), 1177-1184. doi:10.1093/aje/kwt084
Roos, P. M., Vesterberg, O., Syversen, T., Flaten, T. P., and Nordberg, M. (2013). Metal Concentrations in Cerebrospinal Fluid and Blood Plasma from Patients with Amyotrophic Lateral Sclerosis. Biol. Trace Elem. Res. 151 (2), 159-170. doi:10.1007/s12011-012-9547-x

Traxinger, K., Kelly, C., Johnson, B. A., Lyles, R. H., and Glass, J. D. (2013). Prognosis and Epidemiology of Amyotrophic Lateral Sclerosis: Analysis of a Clinic Population, 1997-2011. Neurol. Clin. Pract. 3 (4), 313-320. doi:10.1212/ CPJ.0b013e3182a1b8ab

van Es, M. A., Hardiman, O., Chio, A., Al-Chalabi, A., Pasterkamp, R. J., Veldink, J. H., et al. (2017). Amyotrophic Lateral Sclerosis. The Lancet 390 (10107), 2084-2098. doi:10.1016/s0140-6736(17)31287-4

Verbanck, M., Chen, C.-Y., Neale, B., and Do, R. (2018). Detection of Widespread Horizontal Pleiotropy in Causal Relationships Inferred from Mendelian Randomization between Complex Traits and Diseases. Nat. Genet. 50 (5), 693-698. doi:10.1038/s41588-018-0099-7

Wang, M., Liu, Z., Sun, W., Yuan, Y., Jiao, B., Zhang, X., et al. (2020). Association between Vitamins and Amyotrophic Lateral Sclerosis: A Center-Based Survey in Mainland China. Front. Neurol. 11, 488. doi:10.3389/fneur.2020.00488

Xu, L., Liu, T., Liu, L., Yao, X., Chen, L., Fan, D., et al. (2020). Global Variation in Prevalence and Incidence of Amyotrophic Lateral Sclerosis: a Systematic Review and Meta-Analysis. J. Neurol. 267 (4), 944-953. doi:10.1007/s00415019-09652-y

Zhang, X., Chen, S., Li, L., Wang, Q., and Le, W. (2008). Folic Acid Protects Motor Neurons against the Increased Homocysteine, Inflammation and Apoptosis in SOD1G93A Transgenic Mice. Neuropharmacology 54 (7), 1112-1119. doi:10.1016/j.neuropharm.2008.02.020

Zheng, J.-S., Luan, J. a., Sofianopoulou, E., Imamura, F., Stewart, I. D., Day, F. R., et al. (2021). Plasma Vitamin C and Type 2 Diabetes: Genome-wide Association Study and Mendelian Randomization Analysis in European Populations. Diabetes Care 44 (1), 98-106. doi:10.2337/dc20-1328

Zoccolella, S., Bendotti, C., Beghi, E., and Logroscino, G. (2010). Homocysteine Levels and Amyotrophic Lateral Sclerosis: A Possible Link. Amyotroph. Lateral Scler. 11 (1-2), 140-147. doi:10.3109/17482960902919360

Conflict of Interest: The authors declare that the research was conducted in the absence of any commercial or financial relationships that could be construed as a potential conflict of interest.

Publisher's Note: All claims expressed in this article are solely those of the authors and do not necessarily represent those of their affiliated organizations, or those of the publisher, the editors and the reviewers. Any product that may be evaluated in this article, or claim that may be made by its manufacturer, is not guaranteed or endorsed by the publisher.

Copyright $\odot 2022 \mathrm{Mu}$, Zhao, Han, Tian, Guo, Zhang, Zhu, Zhang, Zhang and Liu. This is an open-access article distributed under the terms of the Creative Commons Attribution License (CC BY). The use, distribution or reproduction in other forums is permitted, provided the original author(s) and the copyright owner(s) are credited and that the original publication in this journal is cited, in accordance with accepted academic practice. No use, distribution or reproduction is permitted which does not comply with these terms. 